\title{
Language of Science and Language for Science: The Way to Sustainable Development in Africa
}

\author{
Abdoulaye Hakibou \\ Littérature et Linguistique Anglaise, Université de Parakou, Parakou, Bénin \\ Email: hakibouabdoulaye@yahoo.fr
}

How to cite this paper: Hakibou, A. (2020). Language of Science and Language for Science: The Way to Sustainable Development in Africa. Advances in Literary Study, 8, 157-166.

https://doi.org/10.4236/als.2020.84013

Received: April 23, 2020

Accepted: August 31, 2020

Published: September 3, 2020

Copyright $\odot 2020$ by author(s) and Scientific Research Publishing Inc. This work is licensed under the Creative Commons Attribution International License (CC BY 4.0).

http://creativecommons.org/licenses/by/4.0/

(c) (i) Open Access

\begin{abstract}
Africa is almost fully known for the great number of its local languages, number diversely appreciated by natives and non-natives, mainly researchers in linguistics. Some take the great number of local languages in individual countries as a handicap to national language use whereas others consider it as a cultural prosperity. Today, the question is about the real linguistic needs for Africa to start science transmission in them for competitive and sustainable development. The researcher believes that African development cannot be effective and sustainable without scientific acquisitions and applications of its own. The present paper aims to discuss the needs through which African languages could be used to conduct instructions and scientific researches. The results of the study show that some effort started with functional literacy classes in rural areas with limited contents and that today, with formal learners from universities in local language literacy classes, the main condition along with linguistic policies is lexical needs to express new cultural realities and conduct scientific writing in local languages as they try them in western languages.
\end{abstract}

\section{Keywords}

Functional Literacy, Abstraction, Lexical Need, Sustainable Development, Scientific Writing

\section{Introduction}

Evidences accounting the contact between Western countries and Africa exist in many domains. The linguistic domain is the most expressive since the colonial languages are still the official mediums of communication in almost all the African countries. What is obvious as the consequence of the contact is that western languages very soon served as the means of intercommunication, mainly in for- 
mal context. The researcher does not consider that contrast as a problem in itself, but rather the incapacity of the elite to use their new acquisitions through western languages as a platform to promote their mother tongue so as to ease scientific acquisitions to future generations for better applications.

At a time in the colonial history, the African elite that started making precision about African realities through literature somehow showed the resistance of African local languages through their productions though they are in western languages, mainly French and English. That spot use of local languages in literature can be considered as a take-off that came to be accompanied with literacy policies in individual countries mainly south of the Sahara and just after the independences. The literacy policy that well started in those countries in its functional phase remained very soon limited to that phase. The stagnation continues until today when new types of learners emerged in literacy classes, demanding the phase of abstraction in local language teaching which the researcher considers as the most important condition to the role of formal instruction that should be assigned to local languages.

It is in that context of local language promotion that the present study is framed and aims to carry out a situational photograph as to show not only the concrete effort made, but also the lexical gap that should be filled in so as to render local languages ready to be used as official medium of instruction in individual countries where linguistic policies wait for effective applications.

The present paper is theoretically based on contact sociolinguistics with a glance at Hudson's process of standardization of language just to recall how we could get standard variety from the many possible ones for most of the local languages concerned in the study. It starts from a situational description that allows the researcher to present effort done mainly in functional literacy before indicating the key steps necessary to effective and efficient use of local languages in formal instruction. The methodological approach of the study is a situational analysis with comments from proximity enquiry in two selected countries. The development of the paper starts with a recall of Hudson's theory about language standardization; it continues with the situational description about effort made in individual countries and ends up with the steps necessary to the formal use of local languages in the countries south of the Sahara.

\section{The Process of Language Standardization}

Languages are in relation with societies and cultures. The creation of varieties of a given language holds to haphazard way, "largely below the threshold of consciousness of the speakers" (Hudson, 1996: p. 32). But, to get a variety that can stand an official one for a general linguistic community, the society intervenes with full awareness and clear objectives. This intervention called 'standardization' goes through a process comprising four essential steps. For any language to be used as an official language, it must be a variety selected by the community and that underwent codification and to which some function has been elabo- 
rated, and that is eventually accepted.

\subsection{Selection}

The very first step of the process of standardization, selection is the fact of choosing a variety that will be developed into a standard language. Most of the time, it is among the existing varieties that one is selected because of a relative prestige it has and that speakers of it share. But, in some cases, it is possible to virtually grind the existing varieties into one that carries relatively characters of the varieties. Once the selection made, the process continues with the codification.

\subsection{Codification}

Codification is the normative step of the process. A structure is created to set norms on the basis of which users of the language will know what is correct or not in the language use. Codification indicates what can be used according to circumstances. Oral production forms and writing production ones should be identified. Every aspect should then be fixed in terms of grammar books and dictionaries. Once codification has taken place, it becomes necessary for any ambitious citizens to learn the correct forms and not to use in writing any other forms that may exist in varieties which have not been selected in the process of standardization. Let's take an example from North Benin for illustration.

In North Benin Baatonum is a very well-known language shared with citizens of Kwara State in the Federal Republic of Nigeria. It has many varieties among which Makaram, Bagum, Bonikparam, Kpabirim and Kpandem are the most easily identifiable. In the seventies, at the very beginning of literacy policy, Makaram was selected as the standard Baatonum. With the progressive codification, no other form, be it item of any of the other varieties is accepted in formal context of writing. This step leads to the acceptance, but codification takes first its force from the elaboration of function.

\subsection{Elaboration of Function}

Elaboration of function is in fact an almost hidden step in the process of standardization. Once a variety is codified, it automatically becomes the one of formality with clearly stated domains and circumstances of use. When a speech community wants to entirely be formal in the process of standardization, it defines those aspects so that any learners should be aware of the different functions assigned to the selected variety. The many functions assigned to a selected variety hold to two circumstances: official affairs of the community and writing contexts that go along with. In many cases of local language development, the functions come along with new lexical needs. In literature for instance, orality is the habit with given form and corresponding vocabulary; going to writing in literature will forcedly demand additional words and expressions not only for internal cohesion but also for international and scientific adequacy. Some technical words are not to be neglected in that context; some can be formed with ex- 
isting words, but new expressions should be coined. Borrowing should help in the process. It is now that acceptance comes in the process.

\subsection{Acceptance}

According to Hudson (1996: p. 33) "the variety has" now "to be accepted by the relevant population as the variety of the community-usually, in fact as a national language". But the most important aspect Hudson (idem) added in that process is development:

Once this has happened, the standard language serves as a strong unifying force for the state, as a symbol of its independence of other states (assuming that its standard is unique and not shared with others), and as a marker of its difference from other states. It is precisely this symbolic function that makes states go to some lengths to develop one.

As our daily struggle is about/for development, African local languages need close attention where it is possible for their internal development as a platform for African sustainable development.

Those four steps are in fact key steps necessary for the standardization of a variety among many of a given language. Though the linguistic problem in Africa south of the Sahara is not quite about standardization, the paper is through it because some steps are still to be revisited for local languages to acquire a form necessary for their official use in governmental policies, mainly education. For, it will not be acceptable that a language chosen for official function be in acute shortage of linguistic items that should help in its new function. The paper also draws the attention of individual speech communities on that aspect in order to have language with sufficient material for use. And that condition brings the researcher to present the state of affairs in local language matters. The study took the example of Benin and Togo for different needs of illustrations.

\section{Situational Analysis of Local Language Promotion}

The study went through interviews with people in selected areas of the two countries. Related to time and mainly to financial means constraints, the number of interviewees is reduced to a reasonable minimum with the characteristic that the selection is guided. The proximity enquiry took into account only people in literacy process in their mother tongue. The work was conducted with forty-eight (48) people of whom only two were women. Four zones were identified, two in Benin and two in Togo. The interviews took place in the North and the south of the two countries. Twelve interviewees were used in each zone. That sample was not taken on the basis of a determined number of literacy actors, but rather a minimum sample to get varied aspects of the issue. It must be notified that only the enquiries conducted in the North of Benin and North of Togo had owners of Bachelor of Art (BA) among the interviewees. It was a coincidence, since the level of instruction was not a criterion of selection. The essential of the contents of the interviews was about general concerns about language policy, the 
local languages in coexistence, literacy effort and constraints, and lexical needs.

\subsection{Local Language Coexistence in Individual Countries}

In Benin and Togo in West Africa, the coexisting languages belong to larger groups. The study went through enumeration of groups and their individual contents: Kwa and Gur are the main language group in existence in the two countries. Kwa is a regional group that goes up to Côte d'Ivoire (Ivory Coast) and Nigeria in West Africa. In Togo and Benin, that group is in the south. It is subdivided into two subgroups: Gbe and Ede. Many languages are in each of the two subgroups. In the Gbe group there are languages like Ewe, Gen, Fon, Gun etc. The Ede group mainly in Benin is from the south-east to the centre of the country and in south-west of Nigeria. The main languages it contains are Yoruba and its many varieties like Itcha, Ife, tchaabe. They have a light inter-intelligibility. Variations and differences are mainly more phonological than semantic.

The second main group after Kwa of the south is the Gur group in the north of Togo and Benin, in Burkina Faso and Nigeria. It contains too many languages in the northern parts of the two countries. In Benin and Nigeria, two common languages belong to the group: Baatonum and Boo. It is in the North-west of Benin that the Gur group contain many languages: Ditamari, Wama, Biali, Gourmantche, Yende, Gangam. The interviewees in the north attested that, in some administrative units, two contiguous villages can have two different languages with no inter-intelligibility. The same realities are mentioned by interviewees in North Togo. There, Kabiye is the most spoken language with many varieties, and then come Kotokoli, Ditamari, Gangam, Gourmantche with varieties and other languages shared with Ghana.

Mutual intelligibility is a phenomenon accounting the fact that two speakers of two different varieties of a language understand one another. This is possible in some countries like Benin and Togo mainly in the Ede and Gbe groups. But in the Gur group, the many languages existing in it have more unique forms than languages with varieties. However, some of them have varieties like Baatonum with five varieties and Ditamari with two.

That linguistic situation needs close attention in individual countries for definition of objective criteria to see where to start from. It is a major concern in linguistic policy elaboration. It is not possible to start with all the languages in coexistence inside the countries. The participants to the study suggested that enumerations be conducted to establish prioritization of main spoken languages in the different language groups evoked in the study. It is through literacy classes that most of the local languages are best described.

\subsection{Literacy Efforts and Constraints in the Process of Local Language Promotion}

Literacy efforts evoked by the interviewees go from policy definition, material design to effective classes. For policy definition, most of the interviewees said 
that literacy matters started with rural area farmers to help them to do calculation, writing and selling their products with bascules. Rural development services where in charge of the implementation. In the case of Benin, the policy started with some languages and progressively with many of them. Some related services were created. Materials like books were designed through workshops and printed in literacy services. Due to the fact that learners are adult and farmers, arranged time tables were established; classes were held in the afternoons only. Effective classes were organized around reading, writing and counting. Designed texts were about their immediate environment and daily activities. Interviewees evoked some constraints in the literacy process.

The very first constraint learners coped with was the fact that learners go to the farm in the mornings and literacy classes in the afternoons. Some participants lacked assiduity and classes held without consistency. The second constraint was that the classes were organised for adults, mainly men. Children were assigned learning in formal school, thus in colonial languages. The third constraint was about the shortage of the materials: documents where not sufficient though they were locally produced. Those constraints were related in many of the countries to the fact that the classes were scheduled for farmers mainly. But, with the new type of literacy class participants, other constraints appeared of which is the need of specific words to express specific realities.

\subsection{New Lexical Needs in Local Languages}

Most of the interviewees declared that literacy class participants are now literate people, that is, people having gone to formal school. Some of the interviewees said that they got Bachelor of Art degree (university) and others gave up school before A-level. So, many of them know report, essay, thesis or dissertation writing. In the interviews, a concern was about the difference between "language of science" and "science language". Answers varied according to the level of instruction of the participant. But the common aspects of their definitions were that any existing language can be worked to be used in science and that specific terminologies in science stand the science language, using linguistic items to mean something in science and something else in the daily use of the language. After individual comments and justification of the difference between language of science and science language, they expressed their new lexical needs to be performant in local language use.

In fact, that aspect of the study was inserted in the interview guide in terms of vocabulary check. A list of words in French was presented to them and the instruction was to say the same thing in their mother tongue. Most of the participants used sentences to translate single words or phrases. They realized that we need simple words and phrases in our languages to express realities we are already accustomed to in western languages. So, the lexical effort is necessary in the promotion of local languages. Some of them found few words in local languages corresponding to the words listed in French. In the discussion, those who 
proposed existing words in their language declared that they matched the meaning of those words to some realities in their culture. So, culture intervenes in word coinage. It is true that lexical items come into language with minimal cultural content and as Zengel (1962) stated it, cultural contents help to more persistently retain words. But, their retaining holds to their use, with stress on writing. This analysis led the researcher to draw a conclusion: total literacy in local languages is with a primary and integral necessity in the light of the objective of the present study. The participants gave relevant examples to support the idea of lexical need.

The most common examples they gave about Africa south of the Sahara are the names of the days of a week and months of the year. The names evoked are Arabic, with phonological changes from area to area. Interviewees in the south used "taatagbe" for Tuesday; those in the north used "atarata" for the same day. In the word "taatagbe" in Fongbe, "gbe" means day and "taata" Tuesday; in Baatonum, "atarata" is also Tuesday with "tarata" being the Arabic "thsalathsaa", meaning the third day of the week and corresponding to Tuesday in Greco-Roman context. And the difference between "thsalathsaa" and "tarata" or "taata" is due to the phonological influence of each local language on the word in almost all the countries south of the Sahara.

The participants added that in some rural areas people use market days to name days. That fact does not annihilate the lexical need in local languages to express realities. It is known to everyone that comparison is not reason; it is all along known with a local common saying that "new rope is woven from the end of the old one". So, without having the pretention to match local languages to western languages, it is reasonable to shed the latter's linguistic light on the former as far as it is possible.

The scientific domain is a domain with a specific language. Of course, there are words in local languages to express scientific realities, but all the realities with existing words known through the colonial school do not have corresponding forms in local languages, at least known forms to language users. So, we need to have words to express realities conceived in colonial languages and even existing words need to be fixed. Word fixation is one of the steps in language perspectives in Africa south of the Sahara.

\section{Steps to Formal Use of African Local Languages}

African local language users recognise the existence of words in their language and at the same time they are aware of the lack of fixation. The fixation is hence capital in the use of standardised languages in the process of their promotion. It is true that the national linguistic policy is transversal in the whole process, but, for the sake of the present reflexion, we have it in the present section of the paper in terms of how to apply it. However, the very first step in the formalisation of local languages in Africa south of the Sahara is the creation of language management institutions for individual languages, kinds of academies for them. 


\subsection{Language Fixation and Language Management Institutions}

Synchronic and diachronic studies of language inform people about the state of language at a given time and through time respectively. Fixation is the decision of admitting new words along with their spelling, pronunciation and meanings. The interviewees think that complementarity is of great importance in its realisation. So, individual users may create forms and contents, and then the institutions should study them in order to have them inserted in the lexicon. Interviewees think that writing gives long life to words even if they undergo modification or rarely used whereas oral use only easily leads words to loss. Some examples have been given and I kept one: in one of the mother tongue of the interviewees, the word "eve" is so rarely used that many speakers of the language, even members of the old generation, do not know it. The researcher investigated to finally get it. The word "eve" means "giniru" in Baatonum, one of the local languages in north Benin. But as many speakers do not know it, they distort the use of the word by making a sentence plus the phrase "the following" day (sisiru). Participants evoked the example of Academy in charge of the fixation of linguistic materials borrowed or simply coined. They gave the case of the French Academy with Larousse being the institution of publication of new materials accepted in French with a history about each new word.

In the light of the French example, the researcher draws the attention of stakeholders on the fact that, having similar institutions can boost the formalisation of local languages. The institutions should be in charge of the edition of documents in which words in the individual languages will be published. It must be noted that effort is being made at individual levels with bilingual wordbooks which cannot for the time being stand as real dictionaries. But they recognised that they are just individual effort. Thus, to have full dictionaries in local languages, regional institutions should create ad hoc committees with specific tasks for word study and fixation. At this point, some interviewees drew the attention of possible stakeholders that things will not quickly move if they wait for the government. They suggested that local initiation starts the mission and that the government may intervene in the process the moment when they realise progression in local language reinforcement. When a local language is built to the size of large use, it is then that its users can expect political decision about its instituting. They suggested that private institutions like Non-Governmental Organisations and other partnerships start the job first, and then the government should come with the linguistic policy.

\subsection{Linguistic Policy in African Countries South of the Sahara}

African countries south of the Sahara have individual policies in linguistic matters. But, in the frame of the local language promotion in those countries, specific policies are necessary for activeness in the process. In their individual laws or constitutions, indications are made about local languages. Interviewees in Benin, for example, said that it is written in their constitutional law that local lan- 
guages should be taught as subject and progressively become medium of formal instruction from primary education to universities. But no edict is elaborate to indicate the conditions, the norms and the institutions to start the application.

Taking into account the number of local languages in individual countries and their places along with their characteristics, interviewees suggested that indications must be made to know which languages to start with. In that context, it will be reasonable that the policy takes into account considerations that could help to ease consensus. An example relevant to the case of Benin could be to consider the main language with an advanced level in its promotion as stated above in the three linguistic groups indicated in the present paper: Ede, Gbe and Gur. It is possible that a group contains languages where mutual intelligibility is too weak, like the Gur group. In such cases, further criteria should be defined to ease the consensus.

Almost all the interviewees in the proximity enquiry among literacy class participants suggested that objectivity be the key character to govern the linguistic policy in order to reach the eventual objective which is to use some local languages in formal instruction in the continent. This should be more effective and efficient for the sustainable development in Africa. No country in the world is known to have developed itself through external culture and language to it. Development is through endogenous culture and language. And in the present state of linguistic realities in Africa, the labour is much deeper than people realise it. It demands a political engagement.

African countries continue with colonial policies in many domains and in their implementation, western powers and institutions accompany individual countries with guidance that gives selective support, support being on fields where the helpers have interest. This is like a business principle that cannot be easily overpowered by African politico-administrative authorities. But, the researcher thinks that it is possible to take up the challenge. The direct implication of it is that authorities should work at it in their political effort to reconsider language matters in the way to development.

\section{Conclusion}

The present study has been about how to bring local languages in African countries south of the Sahara to a usable form in formal instruction. The researcher found that the greatness of the number of local languages is first to be discarded and tackle the issue in terms of lexical effort that should be achieved in individual languages before the policy toward official decision for the use of the main ones in formal instruction.

The paper helped to indicate the path to the lexical reinforcement for individual local languages. In the process, it is suggested that close stakeholders start the lexical fixation without necessary waiting for the government. It is in that frame that the steps of standardisation were evoked in order to be sure that linguistic principles are observed in the process. Institutions should be created to 
manage the fixation of words and their diffusion through existing channels. Government should then intervene to move to the application of its linguistic policy.

In the linguistic policy, political authorities are presented as limited by the selective policy in the accompaniment of Western countries and institutions. In that context, it is suggested that government finds strategies to cope with the linguistic issues of the country in order to have some used in formal instruction. Through formal instruction in some local languages for science transmission with better application, Africa could then start the sustainable development.

The linguistic policies in individual countries south of the Sahara suffer from contingencies that are related to hegemonic wills in the world. The situation is as old as the contact between Europe and Africa. As such, the linguistic issue must be sold with intelligence and abnegation in Africa south of the Sahara. The south-south cooperation may help to bring local languages to lexical consistency. Specialists of the domain of cooperation should study that aspect to propose ways of solving language issues in Africa south of the Sahara.

\section{Conflicts of Interest}

The author declares no conflicts of interest regarding the publication of this paper.

\section{References}

Hudson, R. A. (1996). Sociolinguistics (2nd Edition). Cambridge: Cambridge Textbooks in Linguistics, Cambridge University Press.

Zengel, M. S. (1962) Literacy as a Factor in Language Change. American Anthropologist, No. 64. 